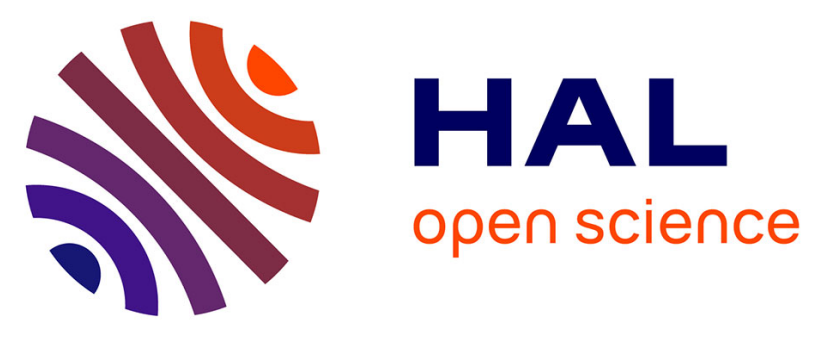

\title{
Quasimonoenergetic electron beams with relativistic energies and ultrashort duration from laser-solid interactions at $0.5 \mathrm{kHz}$
}

Aghapi G. Mordovanakis, James Easter, Natalia Naumova, Konstantin Popov, Paul-Edouard Masson-Laborde, Bixue Hou, Igor Sokolov, Gérard Mourou, Igor V. Glazyrin, Wojciech Rozmus, et al.

\section{To cite this version:}

Aghapi G. Mordovanakis, James Easter, Natalia Naumova, Konstantin Popov, Paul-Edouard MassonLaborde, et al.. Quasimonoenergetic electron beams with relativistic energies and ultrashort duration from laser-solid interactions at $0.5 \mathrm{kHz}$. Physical Review Letters, 2009, 103 (23), pp.235001.1-235001.4. 10.1103/physrevlett.103.235001 . hal-00838486

\section{HAL Id: hal-00838486 \\ https://hal-ensta-paris.archives-ouvertes.fr/hal-00838486}

Submitted on 7 Dec 2015

HAL is a multi-disciplinary open access archive for the deposit and dissemination of scientific research documents, whether they are published or not. The documents may come from teaching and research institutions in France or abroad, or from public or private research centers.
L'archive ouverte pluridisciplinaire HAL, est destinée au dépôt et à la diffusion de documents scientifiques de niveau recherche, publiés ou non, émanant des établissements d'enseignement et de recherche français ou étrangers, des laboratoires publics ou privés. 


\title{
Quasimonoenergetic Electron Beams with Relativistic Energies and Ultrashort Duration from Laser-Solid Interactions at $0.5 \mathrm{kHz}$
}

\author{
Aghapi G. Mordovanakis, ${ }^{1,2, *}$ James Easter, ${ }^{1}$ Natalia Naumova, ${ }^{3}$ Konstantin Popov, ${ }^{4}$ Paul-Edouard Masson-Laborde, ${ }^{5}$ \\ Bixue Hou, ${ }^{1}$ Igor Sokolov, ${ }^{6}$ Gérard Mourou, ${ }^{9}$ Igor V. Glazyrin, ${ }^{7}$ Wojciech Rozmus, ${ }^{4}$ Valery Bychenkov, ${ }^{8}$ \\ John Nees, ${ }^{1}$ and Karl Krushelnick ${ }^{1}$ \\ ${ }^{1}$ Center for Ultrafast Optical Science, University of Michigan, 2200 Bonisteel, Ann Arbor, Michigan, 48109, USA \\ ${ }^{2}$ Department of Physics, University of Toronto, 60 St. George Street, Toronto, M5S 1A7, Canada \\ ${ }^{3}$ Laboratoire d'Optique Appliquée, ENSTA-CNRS-École Polytechnique, F-91761 Palaiseau, France \\ ${ }^{4}$ Department of Physics, University of Alberta, 11322-89 Avenue, Edmonton, Alberta T6G 2G7, Canada \\ ${ }^{5}$ CEA, DAM, DIF, F-91297, Arpajon, France \\ ${ }^{6}$ Space Physics Research Laboratory, University of Michigan, Ann Arbor, Michigan 48109, USA \\ ${ }^{7}$ Russian Federal Nuclear Center-E.I. Zababakhin Institute of Technical Physics, Snezhinsk, Chelyabinsk Region 456770, Russia \\ ${ }^{8}$ Lebedev Physics Institute, Russian Academy of Sciences, Leninskii Prospect 53, Moscow 119991, Russia \\ ${ }^{9}$ Institut de la Lumière Extrême, ENSTA, École Polytechnique, CNRS, 91761 Palaiseau, France
}

(Received 24 April 2009; published 1 December 2009)

\begin{abstract}
We investigate the production of electron beams from the interaction of relativistically-intense laser pulses with a solid-density $\mathrm{SiO}_{2}$ target in a regime where the laser pulse energy is $\sim \mathrm{mJ}$ and the repetition rate $\sim \mathrm{kHz}$. The electron beam spatial distribution and spectrum were investigated as a function of the plasma scale length, which was varied by deliberately introducing a moderate-intensity prepulse. At the optimum scale length of $\lambda / 2$, the electrons are emitted in a collimated beam having a quasimonoenergetic distribution that peaked at $\sim 0.8 \mathrm{MeV}$. A highly reproducible structure in the spatial distribution exhibits an evacuation of electrons along the laser specular direction and suggests that the electron beam duration is comparable to that of the laser pulse. Particle-in-cell simulations which are in good agreement with the experimental results offer insights on the acceleration mechanism by the laser field.
\end{abstract}

DOI: 10.1103/PhysRevLett.103.235001

PACS numbers: $52.38 . \mathrm{Kd}, 52.65 . \mathrm{Rr}$

Relativistic electron beams from laser-plasma interactions can make accessible, in a compact setup, the science and applications that are presently limited to large-scale accelerator facilities. The radiation sources driven by these beams could be instrumental for the study of transient dynamics down to the femtosecond scale in solid-state physics, material science, and biochemistry. Additionally, by virtue of their superior penetration depth in biotissues, electron beams are a promising alternative to $\mathrm{x}$-ray beams for cancer radiotherapy [1]. All these applications would greatly benefit from bright sources of strongly relativistic electrons with a narrow energy spread. However, to date, relativistic and monoenergetic electron beams have been solely produced in under-dense plasmas at low repetition rates [2-4]. While the last few years witnessed considerable efforts to push the beam energy well into the $\mathrm{GeV}$ energy range, little has been done toward achieving higher electron fluxes and beam repetition rates. This is due in part to the difficulty of operating a gas jet - the target of choice for laser-driven accelerators - at high repetition rates, and in part to the substantial amount of laser energy per pulse required to set a wakefield accelerator and induce plasmawave breaking to launch an electron bunch into the accelerator. In this Letter we propose an alternate scheme for laser acceleration at high repetition rate using a bulk glass target $\left(\mathrm{SiO}_{2}\right)$. We demonstrate that, by carefully tuning the plasma density scale length, relativistic electron beams with a relatively narrow energy spread are obtained when the target is excited by tightly focused millijoule-level laser pulses at $0.5 \mathrm{kHz}$. Since their velocities are already comparable to the speed of light, these electron bunches can be subsequently accelerated in a laser-plasma channel-also operating at $\mathrm{kHz}$ repetition rate-that will boost their energy to $>50 \mathrm{MeV}$ preserving their absolute energy spread yielding a monoenergeticity $<1 \%$.

Over the last decade several groups have reported the observation of collimated electron jets produced by $p$-polarized intense laser pulses focused onto solid-density plasmas [5-7]. For obliquely incident laser pulses at nearrelativistic intensities, experiments $[6,8-10]$ and simulations [11-13] have shown that the electron beams are emitted at an angle between the laser specular and the target normal directions and that this emission direction depends critically on the plasma scale length [8]. In most cases the electron spectra were observed to follow Maxwell-like distributions [5,6,9], save for two recent experiments $[14,15]$ where quasimonoenergetic distribution were obtained from pre-exploded foil targets. None of these experiments investigated the electron energy distribution within the collimated jet as a function of the plasma scale length. Here we study both the spatial distribution and the energy spectrum of the electron beam as a function 
of the plasma scale length at laser intensities up to $2.5 \times$ $10^{18} \mathrm{~W} / \mathrm{cm}^{2}$.

The electron experiments were carried out on the $\lambda^{3}$ laser facility at the Center for Ultrafast Optical Science (CUOS). This high-contrast ultrafast system-described in detail elsewhere [16]—produces $30 \mathrm{fs}, 3 \mathrm{~mJ}$ pulses at $0.5 \mathrm{kHz}$ repetition rate. When focused tightly, peak intensities exceeding the relativistic threshold $(\sim 2 \times$ $10^{18} \mathrm{~W} / \mathrm{cm}^{2}$ for $800 \mathrm{~nm}$ wavelength) can be reached in a $1.5 \mu \mathrm{m}$ (FWHM) focal spot. This distinguishes our approach to quasimonoenergetic electron beam generation from all previous relativistic-intensity experiments which were carried out using large laser facilities at low repetition rates. While amplified spontaneous emission (ASE) is incident on the target starting a few nanoseconds before the main interaction, its intensity remains below the damage threshold of $\mathrm{SiO}_{2}$ up until $\sim 20$ ps prior to the peak of the main pulse. During these last $20 \mathrm{ps}$, the ASE is expected to cause plasma formation, but only to produce a very short scale length. Thus, in order to study the electron beam characteristics as a function of the plasma density scale length, we deliberately introduce a moderateintensity prepulse $\left(2 \times 10^{14} \mathrm{~W} / \mathrm{cm}^{2}\right)$ with a variable delay, focused to a much larger spot size $(\sim 30 \mu \mathrm{m})$ compared to that of femtosecond pulse. We relied on a one-dimensional hydrodynamic code (HYADES [17]) to relate the prepulse delay in ps to the scale length in $\mu \mathrm{m}$, assuming an isothermal expansion.

In the typical experimental geometry (Fig. 1), the $p$-polarized pulses were incident at $45^{\circ}$ onto an $\mathrm{SiO}_{2}$ target having an optical-quality surface. The target was translated to ensure that each laser pulse interacted with an undamaged area. The spatial profile of the emitted electron beam

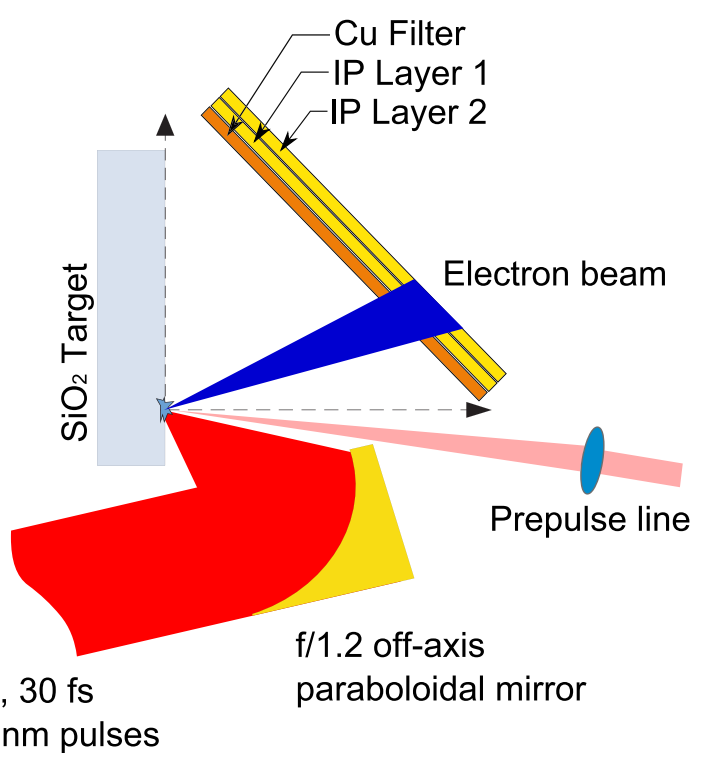

FIG. 1 (color online). Experimental setup used to probe the spatial distribution of the hot electron beam. Alternately, an electron spectrometer is used instead of the IP stack to characterize the electron beam spectrum. was recorded on a stack of imaging plates (IPs) (FUJI BAS-SR 2025, calibrated in [18]) mounted to cover the angular cone between the target surface and normal directions, opposite the laser propagation direction. The first IP layer was covered with a $50 \mu \mathrm{m}$ thick $\mathrm{Cu}$ filter that cuts off electrons with energies below $\sim 150 \mathrm{keV}$. The second layer was filtered by the first IP layer along with the $\mathrm{Cu}$ foil and cuts off $\lesssim 350 \mathrm{keV}$ electrons. The electron spectrum was sampled with a custom-built dispersive spectrometer having an $8 \mathrm{~cm}$-long collimator that reduces the acceptance angle to $\sim 30 \mu \mathrm{rad}$. The collimator was typically aligned along the peak of the electron beam about $5 \mathrm{~cm}$ away from the plasma.

The spatial distribution of the electron beam emitted by the $\mathrm{SiO}_{2}$ plasma was studied as a function of the scale length at an intensity of $\sim 2.5 \times 10^{18} \mathrm{~W} / \mathrm{cm}^{2}$. The prepulse delay $\delta t$ was varied between $0 \mathrm{ps}$ (blocked prepulse) and $187 \mathrm{ps,} \mathrm{corresponding} \mathrm{to} \mathrm{a} \mathrm{scale} \mathrm{length} \mathrm{range} \mathrm{varying}$ between $<0.1 \lambda$ and $\sim 5.5 \lambda$, respectively. The electron signal was recorded on the IP stack in the single-shot operation mode. Figures 2(a) and 2(b) show IP data from the first (left column, lower energies) and the second layer (right column, higher energies) for different prepulse delays. The laser specular half-intensity cone, centered at $45^{\circ}$, is marked with a dashed circle for reference. The target normal and surface directions are oriented along the $0^{\circ}$ and $90^{\circ}$ angles. For short to intermediate scale lengths $\left(L_{n} \lesssim \lambda / 2 ; \delta t \lesssim 16.7 \mathrm{ps}\right)$, the electrons were emitted into a well collimated beam centered along the $34^{\circ}$ direction, between the specular and the normal directions. The integrated electron signal recorded on the IP increased with increasing prepulse delay (scale length) and peaked when $L_{n} \simeq \lambda / 2$. At this optimum scale length, the electron beam divergence was measured to be around $\pm 7^{\circ}$ (for electrons with $E>350 \mathrm{keV}$ ). For longer predelays $(\delta t \geq 20.0 \mathrm{ps}$ ), the electron beam broke up and was emitted over a broader cone. Moreover, the beam direction gradually shifted toward the target normal direction for very long predelays, as would be expected when resonance absorption in a long scale length plasma is dominant. Also observed in Fig. 2 (notably in panels a-i, a-ii, b-i, b-ii) is a hole in the electron beam spatial distribution along the axis of the laser specular beam where the intensity is highest. This suggests that electrons are expelled from the beam by the ponderomotive force of the relativistic-intensity laser. However, such a scenario could only occur if the electron beam is emitted while the laser pulse is reflecting off the target surface. As a consequence, the duration of the electron pulse-at the source-must be less than or comparable to that of the laser pulse, i.e. $\sim 30 \mathrm{fs}$.

In addition, we investigated the energy distribution of the electrons within the beam for various preplasma conditions. The spectra were sampled along the $36^{\circ}$ direction (with respect to the normal) by accumulating 250 $p$-polarized pulses at a peak intensity of $2 \times$ $10^{18} \mathrm{~W} / \mathrm{cm}^{2}$. For short to intermediate scale lengths, the spectra exhibited a non-Maxwellian quasimonoenergetic 


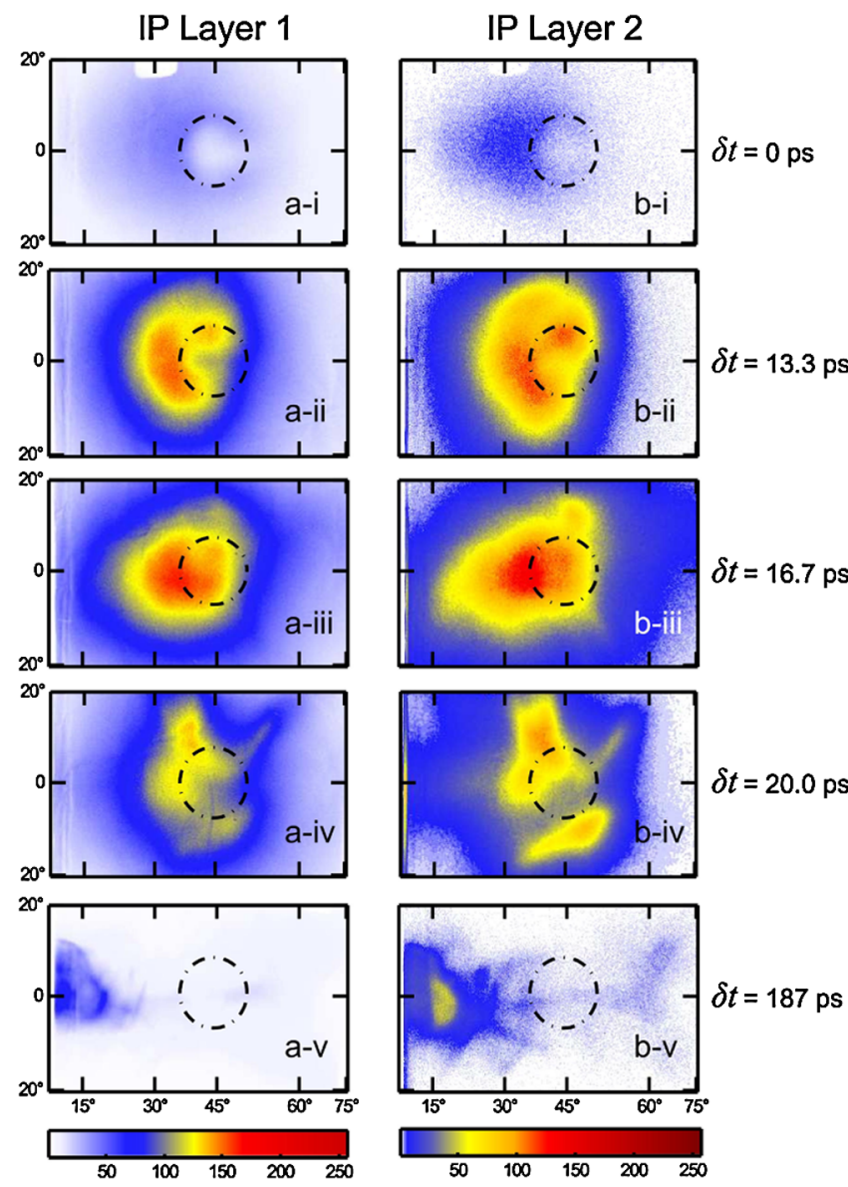

FIG. 2 (color online). Single-shot exposures of the electron beam from $\mathrm{SiO}_{2}$ plasma for different prepulse delays $(\delta t=0$, $13.3,16.7,20.0$ and $187 \mathrm{ps})$. The laser intensity is $2.5 \times$ $10^{18} \mathrm{~W} / \mathrm{cm}^{2}$ incident along the $45^{\circ}$ angle (marked with a dotted circle; the target surface direction is along the $90^{\circ}$ angle). The left and right columns show electrons registered on IP layer 1 $(>150 \mathrm{keV})$ and layer $2(>350 \mathrm{keV})$, respectively.

peak at relativistic energies (Fig. 3). Similarly to the behavior of the beam's spatial distribution, the spectral peak shifted toward higher energies when the scale length was increased from shortest to $L_{n} \simeq \lambda / 2$ (prepulse delay of $16.7 \mathrm{ps}$ ). At this optimum scale length, the spectral peak occurred at $\sim 0.78 \mathrm{MeV}$ [red (medium gray) curve in Fig. 3] and the total charge in the collimated beam is estimated to be $7 \pm 3 \mathrm{pC}$ per laser shot. This yields an integrated electron charge of the order of a $\mathrm{nC}$ per second when the laser is operating at $0.5 \mathrm{kHz}$. The shot-to-shot stability of the electron beam was confirmed by comparing the spectrum from a single laser shot with $L_{n} \simeq \lambda / 2$ (inset in Fig. 3) to the integrated spectrum of 250 shots. For long scale lengths, as the electron beam broke up and shifted to the normal direction (panels a-v and b-v in Fig. 2), far fewer electrons having a Maxwellian distribution were detected along the spectral measurement direction.

In order to understand the mechanisms of electron beam generation we revert to two-dimensional (2D) particle-incell simulations [19]. We consider the interaction when a

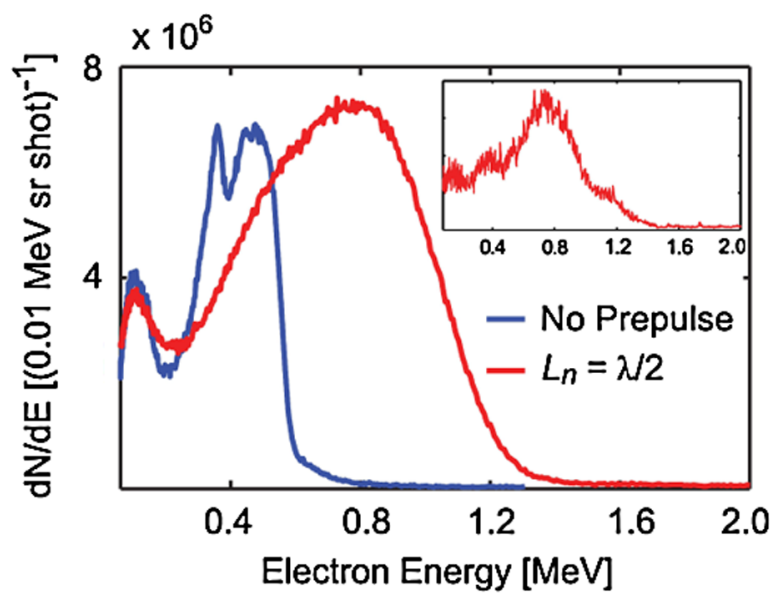

FIG. 3 (color online). Electron spectra measured along the $\phi=36^{\circ}$ direction by accumulating 250 laser shots at $I \sim 2 \times$ $10^{18} \mathrm{~W} / \mathrm{cm}^{2}$ for the case of no prepulse [blue (dark gray)], and a scale length of $\simeq \lambda / 2$ [red (medium gray)]. The inset shows the spectrum from a single laser shot for the optimal scale length of $\sim \lambda / 2$.

30 fs $p$-polarized pulse focused to $2 \lambda$ width with $\sim 2 \times$ $10^{18} \mathrm{~W} / \mathrm{cm}^{2}\left(a_{0}=1\right)$ intensity is incident at $45^{\circ}$ on a collisionless preionized plasma slab with immobile ions and an exponential density profile ranging from $0.1 n_{\text {crit }}$ to a shelf density of $16 n_{\text {crit }}$. Figure 4 shows angular energy distributions of electrons outside the plasma for scale length conditions $L_{n}=\lambda / 5$ and $\lambda / 2,20$ laser cycles after the peak of the pulse has passed through focus. As can be seen in the figure, the particle-in-cell (PIC) simulations reproduce the main features that are observed in experiments: the electrons are bunched in a collimated beam with a quasimonoenergetic distribution, emitted between the laser specular and the target normal directions. Moreover the highest energy beam is obtained when the scale length $L_{n}=\lambda / 2$, producing a distribution that peaks at $\sim 900 \mathrm{keV}$.

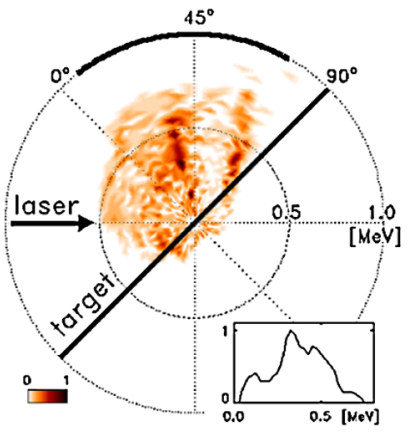

(a)

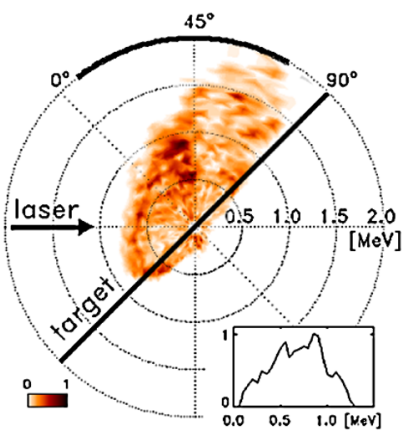

(b)
FIG. 4 (color online). Angular energy distribution of electrons outside the plasma for two different electron density scale lengths: (a) $\lambda / 5$ and (b) $\lambda / 2$. The laser intensity is $\sim 2 \times$ $10^{18} \mathrm{~W} / \mathrm{cm}^{2}\left(a_{o}=1\right)$. The insets show a line plot of the energy distribution along the collimated beam direction. 
Examining the electron trajectories together with evolution of their density and of the fields, we observe the following: when electrons break away from laser-driven oscillations and penetrate deeper into the plasma, a charge separation field is created that drives electrons from the plasma skin layer through the nulls of the fields, where the light pressure is minimal. This process of electron ejection resembles that explained in greater detail in a previous publication [20] at higher laser intensities. Most ejected electrons, which form a beam, originate from the scale length thick region around the relativistic critical density, on the rising edge of the incident pulse intensity, when the superposition of incident and reflected fields exceeds values of $a_{o} \approx 1$. Further, the main energy gain experienced by the ejected electrons occurs as they surf on the field superposition, traversing the pulse as it reflects off the skin-effect region, and veer slightly toward the normal direction, gaining additional energy in the reflected light.

It is also observed in PIC simulations that the reflection of light from shorter scale length plasmas (e.g., $L_{n} \leq \lambda / 2$ ) is sharp and mirrorlike, whereas the reflection through a longer scale length plasma follows a curved path which splits providing a less regular distribution of bunched electrons. This explains the irregular features observed experimentally in the longer scale length cases (panels iv and $v$ in Fig. 2). The optimal regime of electron acceleration from solid targets remains to be studied in greater detail over a wider parameter space.

In another experiment [21], an $\mathrm{Al}$ target was irradiated under similar conditions but yielded qualitatively distinct results: even when no intentional prepulse is present, the electrons were emitted in a much broader and irregular beam with a Maxwellian-like energy distribution. This suggests that the target conductivity may crucially affect electron emission. Al having three electrons in the conduction band behaves similar to a plasma even during the lowintensity ASE preceding the laser pulse. Its lower ionization threshold causes the $\mathrm{Al}$ plasma to initiate expansion at an earlier instant and produces a longer scale length by the time the main pulse reaches the critical region. The $\mathrm{SiO}_{2}$ target, on the other hand, remains un-ionized until the laser field reaches specific ionization thresholds thus producing a shorter scale length plasma. Preliminary simulations using a hybrid code that combines kinetic PIC along with field and collisional ionization modelling show that deeper ionization caused by the strong interacting fields may also contribute to the production of relativistic quasimonoenergetic features in the spectrum by directly injecting the ionized electrons at specific phases of the reflecting fields. These issues will be discussed elsewhere.

To conclude, we demonstrated that by tightly focusing millijoule-energy femtosecond laser pulses onto a solid-density plasma with an intermediate scale length, one can produce collimated beams of relativistic electrons $(\sim 0.8 \mathrm{MeV})$ with ultrafast duration at $\mathrm{kHz}$ repetition rate. By virtue of their relativistic velocities $(\sim 0.92 c)$, these electron bunches can be injected into a second laser-plasma channel operating at the same rate which will accelerate the electron bunches to $\sim 50 \mathrm{MeV}$ while preserving the initial absolute energy spread $(\sim 0.4 \mathrm{MeV})$. Moreover, with the emerging technology of high-averagepower fiber lasers, optical pulses with parameters suitable for the production of such electron beams may soon be available at $>100 \mathrm{kHz}$ repetition rates [22]. This could open the door for relativistic laser-plasma experiments to cross, for the first time, from the laboratory setup to practical applications ranging from novel accelerator concepts to the medical field.

This work was supported by the Army Research Office (ARO) and the National Science Foundation (NSF). I. V. G. and V.B. would like to acknowledge the support of the Russian Foundation for Basic Research. A. G. M. wishes to acknowledge Takeshi Matsuoka for insightful discussions and Professor Kazuo Tanaka for providing imaging plate calibration data.

*aghapi@gmail.com

[1] Y. Glinec et al., Med. Phys. 33, 155 (2006).

[2] S. Mangles et al., Nature (London) 431, 535 (2004).

[3] J. Faure et al., Nature (London) 431, 541 (2004).

[4] C. Geddes et al., Nature (London) 431, 538 (2004).

[5] S. Bastiani et al., Phys. Rev. E 56, 7179 (1997).

[6] D. F. Cai et al., Phys. Plasmas 10, 3265 (2003).

[7] C. Serbanescu and R. Fedosejevs, Appl. Phys. B 83, 521 (2006).

[8] M. I. K. Santala et al., Phys. Rev. Lett. 84, 1459 (2000).

[9] Z. Li et al., Phys. Plasmas 13, 043104 (2006).

[10] Y. T. Li et al., Phys. Rev. Lett. 96, 165003 (2006).

[11] H. Ruhl et al., Phys. Rev. Lett. 82, 743 (1999).

[12] R. Kodama et al., Phys. Rev. Lett. 84, 674 (2000).

[13] Z.-M. Sheng et al., Phys. Rev. Lett. 85, 5340 (2000).

[14] X. Wang, K. Nishikawa, and K. Nemoto, Phys. Plasmas 13, 080702 (2006).

[15] L. M. Chen et al., Phys. Rev. Lett. 100, 045004 (2008).

[16] B. Hou et al., Opt. Express 16, 17695 (2008).

[17] J. T. Larsen and S. M. Lane, J. Quant. Spectrosc. Radiat. Transfer 51, 179 (1994).

[18] K. A. Tanaka et al., Rev. Sci. Instrum. 76, 013507 (2005).

[19] N. Naumova, J. Nees, and G. Mourou, Phys. Plasmas 12, 056707 (2005).

[20] N. Naumova et al., Phys. Rev. Lett. 93, 195003 (2004).

[21] A. G. Mordovanakis et al., Appl. Phys. Lett. (to be published).

[22] J. Limpert et al., IEEE J. Sel. Top. Quantum Electron. 15, 159 (2009). 